\title{
ASPECTOS DA ALIMENTAÇÃO NATURAL DO CAÇÃO- FRANGO Rhizoprionodon lalandii (VALENCIENNES,1841) (ELASMOBRANCHII, CARCHARHINIDAE) NO MUNICÍPIO DE BARRA VELHA, SANTA CATARINA.
}

\author{
LIMA, G.H.L.*; DAROS, F.A.; MAZZOLENI, R. \& M. HOSTIM-SILVA \\ Universidade do Vale do Itajaí. Centro de Ciências Tecnológicas da Terra e do Mar \\ Rua Uruguai, 458. CEP 88302-202, Itajaí - SC, Brasil \\ e-mail: rmazzoleni@cttmar.univali.br
}

\section{RESUMO}

\begin{abstract}
O cação-frango Rhizoprionodon lalandii (Valenciennes,1841) ocorre na costa leste da América do Sul, do Panamá até Santa Catarina. O presente trabalho estudou alguns aspectos do comportamento alimentar de $R$. lalandii. No período de Setembro de 1999 a Junho de 2000 foram coletados mensalmente os indivíduos capturados pela pesca artesanal em Barra Velha, SC. Os itens alimentares foram identificados ao menor nível taxonômico possível. O percentual de ocorrência dos itens foi obtido através da equação: $n .^{\circ}$ de estômagos que continham o item / $\mathrm{n} .^{\circ}$ de estômagos que continham alimento x 100. Dos 78 estômagos examinados, 61 (78\%) continham alimento. Os itens predominantes nos estômagos foram os Teleostei, representando $53 \%$ do total de itens, seguidos pelos Cephalopoda (26\%). A maioria dos teleósteos encontrados no conteúdo estomacal pertencia à ordem Clupeiformes e todos os Cephalopoda pertenciam à subordem Myopsida. O maior valor do grau de repleção (GR) foi igual a GR 4 e a maioria dos indivíduos apresentavam estômagos pouco cheios (GR 2 e GR 1) e alguns continham estômagos vazios $(22 \%)$. R. lalandii, alimenta-se principalmente de espécies pelágicas sendo principalmente pequenos teleósteos e lulas. Como houve uma diversidade baixa de presas, supõe-se que $R$. lalandii seja um predador bastante seletivo.
\end{abstract}

Palavras-Chaves: Rhizoprionodon lalandii, alimentação natural, Sul do Brasil.

\section{NATURAL FEEDING HABITS OF THE BRAZILIAN SHARPROSE SHARL Rhizoprionodon lalandii (VALENCIENNES, 1841) (ELASMOBRANCHII, CARCHARHINIDAE) AT BARRA VELHA, SC, BRAZIL}

\begin{abstract}
ABSTRA CT
The brazilian sharpnose shark Rhizoprionodon lalandii (Valenciennes,1841) occurs in the east coast of South America, from Panama to Santa Catarina. The present work studied some aspects of the feeding behavior of $R$. Ialandii. Samples were collected monthly, between September 1999 and June 2000 being captured by artisanal fleet. The alimentary items were identified to the lowest taxonomic level possible. The occurrence percentage of the items was obtained through the equation: $\mathrm{n} . .-$ of stomachs that contained the item / $\mathrm{n} . .0$ of stomachs that contained food $\mathrm{x} 100$. From the 78 examined stomachs, $61(78 \%)$ contained food. The predominant items in the stomachs were Teleostei, representing $47 \%$ of the total of items followed by Molusca: Cephalopoda (26\%). Most of the bony fishes found in the stomachs content were Clupeiformes and all Cephalopoda represented by the suborder Myopsida. The largest repletion GR 4, most of the individuals presented stomachs filled less than GR 2 and GR 1 , and some had empty stomachs (22\%). R. lalandii, feeds
\end{abstract}

\footnotetext{
*In memoriam
} 
mainly on pelagic species specially small bony fishes and squids. As the diversity of preys was small, it is supposed that $R$. lalandii is a selective predator.

Key Words: Rhizoprionodon lalandii, natural feeding, Southern Brazil.

\section{INTRODUÇÃO}

Carnívoros por excelência, os tubarões compartilham os ápices das cadeias alimentares em ambientes marinhos. Muitos possuem uma dieta oportunista se alimentando desde organismos mortos até animais maiores do que eles próprios. Outros possuem uma dieta altamente seletiva se alimentando de um número reduzido de tipos de presas. Em geral, os tubarões predam os indivíduos mais fracos e doentes das populações de presas, por estes serem mais facilmente capturados.

No Brasil, o conhecimento sobre elasmobrânquios costeiros é ainda incipiente. Entretanto, se comparado às informações disponíveis sobre os tubarões oceânicos ou mesmo do talude continental, os dados existentes são relativamente melhores (Lessa et al, 1998), mas trabalhos sobre alimentação ainda são poucos (e. g. Capitoli et al., 1995; Muto et al., 1995; Vaske, Jr et al., 1993; Vaske \& RincónFilho, 1998).
O gênero Rhizoprionodon possui sete espécies e duas ocorrem no Brasil, R. porosus e $R$. lalandii, habitando águas rasas do litoral e ao redor de ilhas, sendo os tubarões de pequeno porte mais comumente pescados nas praias e baias do litoral brasileiro. Possuem certa importância comercial nos mercados de pequenas cidades litorâneas e sua carne é bastante apreciada (Figueredo, 1977 e Compagno, 1988).

Rhizoprionodon lalandii é uma espécie de ampla distribuição e representatividade nas águas costeiras brasileiras e apresenta uma carência de estudos principalmente relacionados a sua biologia. É o cação mais comumente capturado pela pesca artesanal no Estado de Santa Catarina (R. C. Mazzoleni, obs. pes.) e também freqüentemente capturado pela pesca industrial do mesmo Estado (Kotas, et al, 1995; Mazzoleni \& Schwingel, 1999).

O presente trabalho objetivou estudar alguns aspectos da alimentação natural de Rhizoprionodon lalandiivisando identificar quais

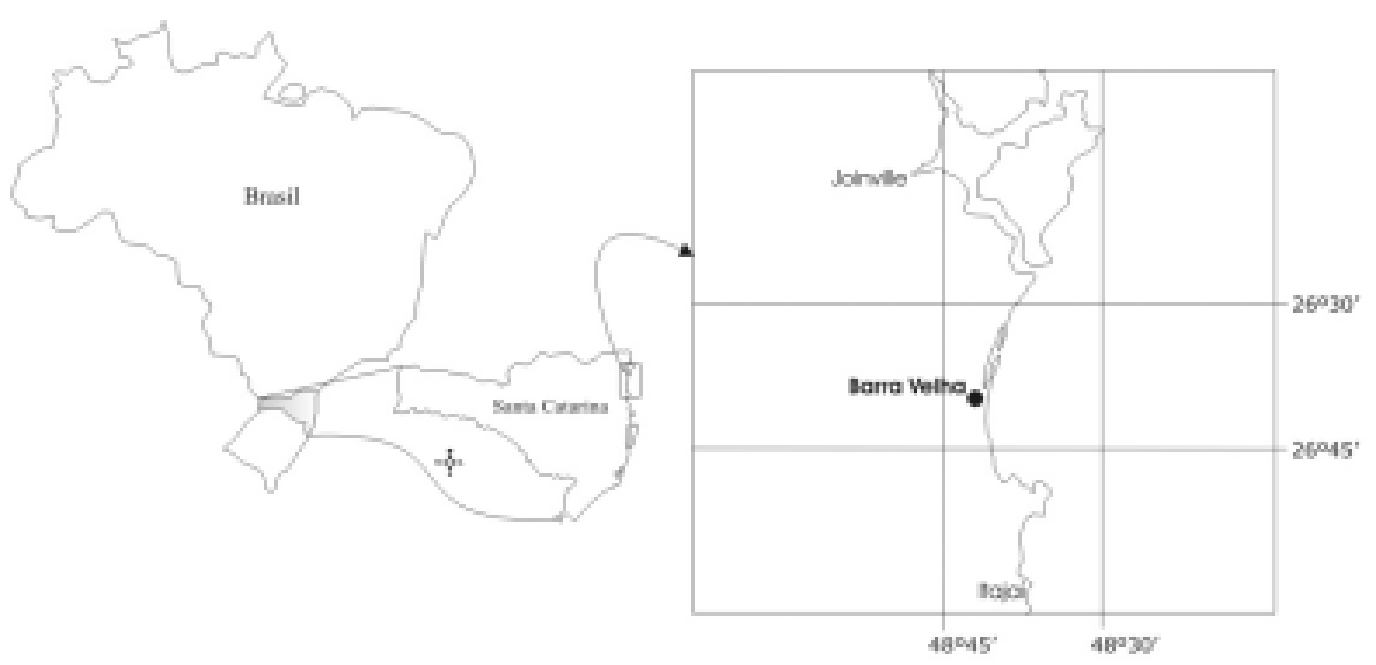

Figura 1 - Localização geográfica da área de estudo 
as presas mais freqüentes e se existe preferência por alguma delas.

\section{METODOLOGIA}

No período de Setembro de 1999 a Junho de 2000 foram coletados mensalmente os indivíduos de Rhizoprionodon lalandii capturados pela pesca artesanal com desembarque na praia de Barra Velha (Figura 1), litoral centro-norte de Santa Catarina, entre as latitudes 2630'S -4830'W e 2645'S - 4845'W. O esforço amostral fora de no mínimo um dia ao mês e procurou-se coletar todos os indivíduos capturados pela pesca neste dia.

Os pescadores desta localidade utilizam em geral redes de emalhe de fundo com malha $7 \mathrm{~cm}$. A área de pesca é compreendida entre as latitudes de 2627'S a 2647'S, alcançando a isóbata dos 30 metros.

Os espécimes capturados foram conduzidos a laboratório, medidos seu comprimento total (CT) tendo seus estômagos retirados para análise. Os conteúdos foram analisados no momento da retirada e apenas o material de identificação duvidosa foi acondicionado em formalina $10 \%$ para posterior identificação. Os itens alimentares foram identificados ao menor nível taxonômico possível. O percentual de ocupação (grau de repleção = GR) foi estimado através de observação macroscópica, GR $1=$ $0-25 \%$; GR 2 = $26-50 \%$; GR $3=51-75 \%$; GR $4=76-100 \%$, e o percentual de ocorrên- cia dos itens pela equação: $n .^{\circ}$ de estômagos que continham o item / n. ${ }^{\circ}$ de estômagos que continham alimento $\times 100$. Para análise, foram agrupados em 3 classes de comprimento (30 a $45 \mathrm{~cm}$.; 45 a $60 \mathrm{~cm}$. e 60 a $75 \mathrm{~cm}$. de comprimento total). Apenas para a determinação do grau de repleção estomacal, decidiu-se aumentar o número de classes de comprimento a fim de permitir uma melhor visualização e interpretação dos dados.

\section{RESULTADOS E DISCUSSÃO}

Foram coletados durante o estudo 79 indivíduos de todos os estágios de maturação (Tabela 1). Foram analisados 78 estômagos, sendo que 61 (78\%) continham alimento. Os itens predominantes nos estômagos foram os teleósteos, representando $54,1 \%$ do total de itens. Muitos estômagos continham Cephalopoda (26\%) e apenas um crustáceo (Ordem Decapoda) foi observado no estômago de um neonato, representando $1,6 \%$ do total de itens (Tabela 2). A maioria dos teleósteos encontrados no conteúdo estomacal pertencia à ordem Clupeiformes, com 6,56\% do total de itens, sendo que destes, 1,6\% pertencia a $\mathrm{Fa}$ mília Engraulidae. Outros grupos de teleósteos identificados foram as famílias Sciaenidae e Trichiuridae, ambas com 1,6\% de ocorrência. Outros itens Teleostei (42,6\%) não puderam ser identificados devido ao alto grau de digestão em que se apresentavam. Todos os itens

Tabela 1 - Número de exemplares amostrados pelo seu estágio de maturação e classe de comprimento total (CT). N $\mathrm{NN}=$ Número de neonato, $\mathrm{N} \mathrm{M}=$ Número de machos adultos, N F = Número de fêmeas adultas.

\begin{tabular}{cccccccc}
\hline \hline Mês & N NN & N M & N F & N 30 - 45 & N 46 - 60 & N 61 - 75 & Total \\
\hline Set/99 & 26 & 0 & 0 & 26 & 0 & 0 & 26 \\
Out/99 & 7 & 0 & 0 & 7 & 0 & 0 & 7 \\
Nov/99 & 9 & 0 & 0 & 6 & 3 & 0 & 9 \\
Dez/99 & 5 & 0 & 1 & 5 & 1 & 0 & 6 \\
Jan/00 & 0 & 1 & 3 & 0 & 4 & 0 & 4 \\
Fev/00 & 0 & 1 & 2 & 1 & 1 & 1 & 3 \\
Mar/00 & 0 & 2 & 14 & 0 & 6 & 10 & 16 \\
Abr/00 & 0 & 5 & 3 & 0 & 8 & 0 & 8 \\
\hline \hline
\end{tabular}


Tabela 2 - Classificação taxonômica e percentuial de ocorrência dos itens alimentares de Rhizoprionodon lalandii

\begin{tabular}{|c|c|c|}
\hline Itens Alimentares & $\overline{\mathbf{N}}$ & Percentual de ocorrência \\
\hline \multicolumn{3}{|l|}{ Filo Arthropoda } \\
\hline \multicolumn{3}{|l|}{$\begin{array}{l}\text { Subfilo Crustacea } \\
\text { Classe Malacostraca }\end{array}$} \\
\hline Ordem Decapoda & 1 & 1,64 \\
\hline \multicolumn{3}{|l|}{ Filo Mollusca } \\
\hline \multicolumn{3}{|l|}{ Classe Cephalopoda } \\
\hline Subordem Myopsida & 16 & 26,23 \\
\hline \multicolumn{3}{|l|}{ Filo Chordata } \\
\hline $\begin{array}{l}\text { Grade Teleostomi } \\
\text { Classe Actinipterygii } \\
\text { Ordem Clupeiformes }\end{array}$ & & \\
\hline Familia Engraulidae & 1 & 1,64 \\
\hline ñ definida & 3 & 4,92 \\
\hline \multicolumn{3}{|l|}{ Ordem Perciformes } \\
\hline Família Scianidae & 1 & 1,64 \\
\hline Trichiuridae & 1 & 1,64 \\
\hline ñ definida & 26 & 42,62 \\
\hline Não identificados & 22 & 36,07 \\
\hline TOTAL & 61 & \\
\hline
\end{tabular}

Cephalopoda identificados eram lulas (subordem Myopsida).

Os teleósteos foram a maioria dos itens em todas as classes de comprimento e o grupo de maior freqüência (Figura 2). Nota-se um incremento na proporção de lulas na dieta de $R$. lalandii conforme estes crescem, sendo a freqüência numérica $18,2 \%$ na classe de $30 \mathrm{a}$ $45 \mathrm{~cm}$ de comprimento total, $33,3 \%$ na classe de 45 a $60 \mathrm{~cm}$ de comprimento total e $50 \%$ na classe de 60 a $75 \mathrm{~cm}$. de comprimento total, igualando-se a proporção de peixes teleósteos capturados nesta última classe de comprimento (Figura 2). Provavelmente o cação-frango, a medida que cresce, utiliza as lulas como uma presa importante na manutenção de suas necessidades metabólicas. O aumento na proporção de lulas no conteúdo estomacal de indivíduos de maior porte pode estar relacionado com uma maior eficiência de predação adquirida por estes. Por outro lado, os indivíduos de menor tamanho parecem possuir uma dieta mais ampla, e exercendo uma procura menor por Cephalopoda.

De acordo com os resultados obtidos, R.lalandii parece preferir se alimentar de pequenos teleósteos e lulas. Estes resultados não estão totalmente de acordo com Figueredo (1977), pois este observou que $R$. Ialandii alimenta-se principalmente de crustáceos e pequenos peixes como sardinhas e manjubas. Porém, para obter informação correta de seletividade, seria necessário fazer observações diretas ou experimentais, em que se colocam à disposição do animal duas ou mais presas em iguais condições de serem escolhidas ou obter informações sobre a dieta na natureza por longos períodos (Zavala-Camin, 1996). 
Os maiores graus de repleção atingiram o GR 4. A maioria 51,3\% $(n=40)$ dos indivíduos apresentavam estômagos pouco cheios (GR 2 e GR 1) e 21,97\% continham estômagos vazios ( $n=17)$. Segundo Zavala-Camin (op cit), a saciação em peixes geralmente ocorre antes de o estômago se encontrar completamente cheio.

A maior média de graus de repleção foi encontrada em indivíduos na classe de 55 a 60 centímetros de comprimento total (53\%). Os indivíduos de menor porte, geralmente neonatos, apresentaram volumes de repleção menores do que indivíduos maiores, como juvenis e adultos (Figura 3), excluindo-se fêmeas grávidas.

É importante ressaltar que é relativamente comum a ocorrência de regurgitação em tubarões enquanto lutam para livrarem-se da captura e a grande ocorrência de estômagos pouco cheios ou vazios pode ter sido dada devido a este fator.

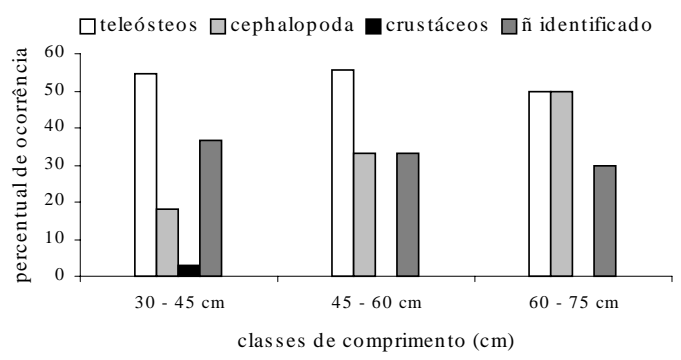

Figura 2 - Percentual de ocorrência dos itens alimentares por classe de comprimento total

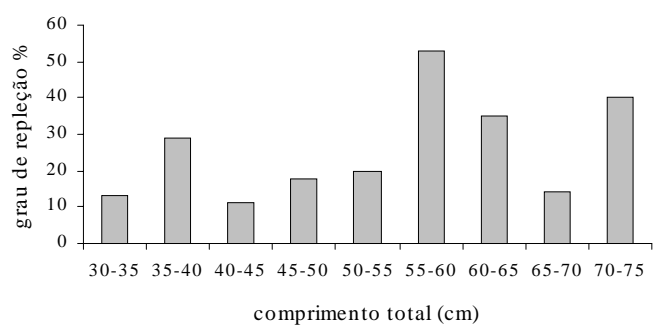

Figura 3 - Graus de repleção por classes de comprimento total
Nenhuma fêmea grávida observada continha alimento no estômago. Pode-se inferir que isto aconteça devido ao fato das fêmeas deixarem de se alimentar durante a gravidez $(\mathrm{H}$. L. Pratt, com. pes.) ou talvez, as fêmeas grávidas podem ser mais suscetíveis à regurgitação causada pelo estresse da captura.

\section{CONSIDERAÇÕES FINAIS}

Rhizoprionodon lalandii, alimenta-se principalmente de espécies pelágicas sendo principalmente pequenos peixes ósseos e lulas. Apesar de ainda inconclusivo, sugere-se que a baixa riqueza de presas seja um indicativo para considerarmos $R$. lalandii como um predador bastante seletivo. Esta predação ainda mostrou-se diferenciar levemente com o desenvolvimento do indivíduo, o que pode estar relacionado a uma maior eficiência de predação adquirida por estes indivíduos de acordo com o seu tamanho.

\section{AGRADECIMENTOS}

À toda equipe do Laboratório de Ciências Ambientais da UNIVALI pelo auxílio no processamento do material; ao Professor Ricardo Corbetta pelas sugestões e incentivo; ao funcionário João Severino pelo apoio nas saídas de campo; ao Oc. Áthila Bertoncini pela parte gráfica final e principalmente aos pescadores de Barra Velha pela enorme colaboração com a equipe da UNIVALI.

\section{REFERÊNCIAS BIBLIOGRÁFICAS}

Capitoli, R.R.; Ruffino, M.L. \& C.M. Vooren. 1995. Alimentação do tubarão Mustelus schmitti Springer na plataforma costeira do estado do Rio Grande do Sul, Brasil. Atlântica, Rio Grande 17: 109-122 p.

Compagno, L.J.V. 1988. Sharks of the Order Carcharhiniformes. Princeton University Press. Princeton, New Jersey.

Figueredo, J.L. 1977. Manual de peixes marinhos do Sudeste do Brasil, Part. 1, Intro- 
dução, cações raias e quimeras. Museu de Zoologia da Universidade de São Paulo. São Paulo,.

Kotas, J.E., Gamba, M.R. Conoly, P.C., Hostim-Silva, M., Mazzoleni, R.C. \& J.P. Pereira. 1995. A Pesca de emalhe direcionada aos Elasmobrânquios com desembarques em Itajaí e Navegantes/SC. In: Reunião Do Grupo De Trabalho E Pesquisa De Tubarões E Raias Do Brasil, VII, de 20 a 24 de novembro de 1995, Rio Grande. Resumos da VII Reunião do Grupo de Trabalho e Pesquisa de Tubarões e Raias no Brasil. Rio Grande: Fundação Universidade do Rio Grande.

Lessa, R.; Santana, F.M.; Rincón, G.; Gadig, O.B.F. \& A.C.A. El-Deir. 1998. Avaliação a Ações Prioritárias para a Conservação de Biodiversidade da Zona Costeira e Marinha. Biodiversidade de Elasmobrânquios do Brasil. [online] Disponível em http:// www.bdt.org.br/workshop/costa/elasmo/ acesso em novembro.

Mazzoleni, R.C. \& P.R. Schwingel. 1999. Elasmobranch Species Landed in Itajaí Harbor, Southern Brazil. Notas Tec. FACIMAR. 3:111-118.
Muto, E.Y.; Soares, L.S.H. \& R. Goitein. 1995. Alimentação das Raias Raja agassizi e Psammobatis glansdssimilis (Chondrichthyes: Rajidae) da Região Costeira de Ubatuba (SP). XI Encontro Brasileiro de Ictiologia. Resumos. p. I11.

Vaske Jr., T.; Hazin, F.H.V.; El-Deir, A.A.C; Bezerra Jr., J.L. \& C.E. Boeckmann-Vieira. 1993. Análise do conteúdo estomacal dos principais tubarões pelágicos capturados com espinhel no Atlântico Sudoeste Equatorial. VI Reunião do Grupo de Trabalho sobre Pesca e Pesquisa de Tubarões e Raias no Brasil. Resumos. Recife, PE p.37. Vaske Jr. T. \& G. Rincón-Filho. 1998. Conteúdo estomacal dos tubarões azul (Prionace glauca) e anequim (Isurus oxyrinchus) em águas oceânicas no sul do Brasil. Rev. Brasil. Biol., 58(3):445-452.

Zavala-Camin, A. Guia para Estudo da Alimentação Natural em Peixes Marinhos. CNPQ. Maringá: UEDEN; São Paulo: SBI. 1996. 